\title{
Reduction of radial-head subluxation in children by triage nurses in the emergency department: a cluster-randomized controlled trial
}

\author{
Andrew Dixon MD, Chantalle Clarkin MScN, Nick Barrowman PhD, Rhonda Correll HBScN, \\ Martin H. Osmond MD, Amy C. Plint MD
}

\begin{abstract}
Background: Radial-head subluxation is an easily identified and treated injury. We investigated whether triage nurses in the emergency department can safely reduce radial-head subluxation at rates that are not substantially lower than those of emergency department physicians.

Methods: We performed an open, noninferiority, cluster-randomized control trial. Children aged 6 years and younger who presented to the emergency department with a presentation consistent with radial-head subluxation and who had sustained a known injury in the previous 12 hours were assigned to either nurse-initiated or physician-initiated treatment, depending on the day. The primary outcome was the proportion of children who had a successful reduction (return to normal arm usage). We used a noninferiority margin of $10 \%$.
\end{abstract}

Results: In total, 268 children were eligible for inclusion and 245 were included in the final analysis. Of the children assigned to receive physician-initiated care, $96.7 \%(117 / 121)$ had a successful reduction performed by a physician. Of the children assigned to receive nurse-treatment care, $84.7 \%(105 / 124)$ had a successful reduction performed by a nurse. The difference in the proportion of successful radial head subluxations between the groups was $12.0 \%$ (95\% confidence interval [Cl] $4.8 \%$ to $19.7 \%$ ). Noninferiority of nurse-initiated radial head subluxation was not shown.

Interpretation: In this trial, the rate of successful radial-head subluxation performed by nurses was inferior to the physician success rate. Although the success rate in the nurseinitiated care group did not meet the noninferiority margin, nurses were able to reduce radial head subluxation for almost $85 \%$ of children who presented with probable radialhead subluxation. Trial registration: Clinical Trials.gov, no. NCT00993954.
$\mathrm{R}$ adial-head subluxation is a common arm injury among young children and often results in a visit to the emergency department. ${ }^{1}$ This type of injury occurs when forceful longitudinal traction is applied to an extended and pronated forearm. ${ }^{2}$ Radial-head subluxation is easily recognized by its clinical presentation and can be treated by a simple reduction technique involving hyperpronation or supination and flexion of the injured arm. ${ }^{3-7}$

Despite the ease of diagnosis and treatment, children with radial-head subluxation often wait hours in the emergency department for a reduction that takes minutes to perform. ${ }^{8}$ These visits have direct health care costs and involve time and stress for the child and their family. Early treatment and shorter wait times correlate with patient satisfaction. ${ }^{910}$ Patient satisfaction is comparable when minor injuries are cared for by a nurse instead of by a physician..$^{11-13}$ Nurse-initiated treatments are increasingly a focus of health care. ${ }^{14-17}$
Treatment of radial-head subluxation is an appropriate area to consider nurse-initiated care. Our objective was to determine whether triage nurses, trained in the recognition and treatment of radial-head subluxation, could successfully reduce radial-head subluxation at a rate similar to that of physicians.

\section{Methods}

\section{Study design}

We performed an open (i.e., unblinded) noninferiority, cluster-randomized control trial to assess whether triage nurses in the emergency department could, using a medical directive (Appendix 1, available at www.cmaj.ca/lookup /suppl/doi:10.1503/cmaj.131101/-/DC1), achieve rates of successful reduction of radial-head subluxation that were not substantially lower than those of physicians. Unpredictable patient acuity and volume in the emergency department made
Competing interests: None declared.

The preliminary results of this trial have been presented at the Pediatric Academic Societies annual conference in Denver, Colorado in May 2011 and published in abstract form.

This article has been peer reviewed.

Correspondence to: Andrew.Dixon@Alberta

HealthServices.ca

CMAJ 2014. DOI:10.1503 /cmaj.131101 
patient-level randomization unfeasible. In our cluster-randomized design, patients were assigned to receive either nurse- or physician-initiated care based on the day of presentation (cluster).

This study was approved by the Children's Hospital of Eastern Ontario (CHEO) research ethics board. The board deemed that written, informed consent was unnecessary because this study assessed a practice change. On nurse-initiated care days, verbal consent was obtained from the child's parents before reduction was attempted.

\section{Study setting and participants}

All children who presented to the CHEO emergency department with a presumed diagnosis of radial-head subluxation from October 2009 to 2010 were screened for enrolment. The Children's Hospital of Eastern Ontario is a tertiarycare pediatric hospital with 68000 visits for radial-head subluxation annually. There are about 60 triage nurses, all who have at least 2 years' experience, attend a 4-hour triage orientation and complete three 4-hour triage training shifts. Physician coverage includes 26 full-time pediatric physicians trained in emergency medicine, 18 part-time staff physicians with other training (general pediatrics, emergency medicine, family practice) and medical trainees. To ensure comparability of the groups, only children who met the criteria for the medical directive (Appendix 1) were eligible for inclusion. Children who, by history, had a spontaneous reduction of a radial-head subluxation before assessment were not eligible.

\section{Medical directive and training of nurses}

The medical directive permitted triage nurses to attempt a reduction for children who presented with signs and symptoms compatible with radial-head subluxation. Children were eligible for the medical directive if they met the following criteria: aged 6 years or less, findings of a physical exam consistent with radial-head subluxation (not using the affected limb, holding the elbow in extension or slight flexion, forearm in hyperpronation and distressed only on elbow movement) and a known injury within the preceding 12 hours (including but not limited to pulls and falls). Children who met any of the following criteria were excluded: an unknown injury mechanism, clavicle or arm deformity, elbow or wrist swelling, tenderness on palpation, metabolic bone disease, neuromuscular disorder or concern for abuse or neglect.

We used a multimodal training initiative for education about the medical directive. Education included a video featuring reduction techniques, a lecture on the physiology and presentation of radial-head subluxation, and a hands-on, 1-hour training session led by a study investigator or the study coordinator. Training involved instruction on the hyperpronation and supination-flexion methods..$^{47}$ Nurses did not perform reductions until all training was completed. The directive instructed nurses to use hyperpronation for the first reduction attempt, and then the supination-flexion if a second attempt was necessary, given evidence that hyperpronation may be more successful. ${ }^{18}$

\section{Study protocol}

A computer-generated randomization calendar schedule was prepared in advance for the entire study period by a statistician who was not involved in recruitment. The sequence was composed of randomly permuted blocks of 8 . At the beginning of each week, the assignment for each day (i.e., "nurse-initiated treatment" or "physician-initiated treatment" was posted in the emergency department; the day assignment was concealed from the investigators and emergency department staff until this time.

On nurse-initiated treatment days, the triage nurse identified eligible children. The nurse prospectively collected data on the child's age, sex, arm injured and time and mechanism of injury using a standardized form. The nurse then attempted a reduction using hyperpronation..$^{47}$ If the child did not use their arm normally within 10 minutes, a second reduction was attempted via supination-flexion. ${ }^{47}$ If the child still was not using their arm normally, they were placed in the queue to see the emergency department physician, as per their triage time and category. If the nurse-initiated reduction was successful, the nurse recorded the time of normal arm usage, and a physician confirmed normal arm function before discharge.

On physician-initiated treatment days, children with presumed radial-head subluxation were identified by either the triage nurse or treating physician. Children were placed in the queue to see the emergency department physician based on their triage time and category. Their care was managed via usual practice in the emergency department by either a trainee or staff physician. The type or order of reduction manoeuvre was not standardized. The physician recorded the time of normal arm usage and the reduction method used.

We extracted the final diagnosis, discharge time and investigations ordered from the emergency department record. A daily review of charts was used to determine how many children had a final diagnosis of radial-head subluxation during the study period, how many of these had been enrolled in the study and, of those not enrolled, how many met the eligibility criteria for the medical directive. 


\section{Outcomes}

The primary outcome was the proportion of patients whose radial-head subluxation was successfully reduced (defined as normal arm use) by the allocated nurse or physician. Secondary outcomes included length of stay in the emergency department, time to normal arm usage following presentation, proportion of children who had a reduction attempted and for whom a fracture was subsequently diagnosed, and the proportion of patients with radial-head subluxation not identified by the triage nurse. Other outcomes included the proportion of children who underwent radiography, change in nursing success rate over 3month blocks and the successful reduction technique used.

\section{Statistical analysis}

Although this was a cluster-randomized trial, the anticipated number of radial-head subluxation cases per day was less than 1 , therefore the reduction of power due to clustering was expected to be inconsequential, and we did not include a variance inflation factor in the sample size calculation. Previous studies have reported that success rates for first reduction attempts are 74\%-90\%, and that $99 \%$ of radial-head subluxations are reduced by the time of discharge from the emergency department. ${ }^{1,46,8}$

We conservatively estimated that the reduction success rate for physicians would be $90 \%$. Using a success rate of $90 \%$, a noninferiority margin of $10 \%$, type I error of 0.05 , and $80 \%$ power, 112 patients were required per study arm. Although noninferiority drug trials often use a margin of $2.5 \%-5 \%$, we felt that $10 \%$ was appropriate for this trial because a success rate of $10 \%$ is acceptable to clinicians, according to an informal electronic survey conducted at 3 Canadian pediatric emergency departments. Clinicians agreed that if nurses were capable of safely reducing radial-head subluxation at success rates within $10 \%$ of physician rates, they would consider implementing this protocol at their institution. Nurse-initiated treatment did not preclude children receiving the goldstandard (physician) treatment.

We used per-protocol analysis (i.e., every enrolled child who had a reduction attempted by the care provider indicated by the randomization day was included in the analysis). If the per-protocol analysis indicated noninferiority, an intention-totreat analysis would also be conducted. ${ }^{19,20}$

A priori, we defined children whose parents did not provide consent for a nurse to attempt the reduction as having had a failed nurse-initiated reduction. (However, no parent refused consent). We considered 2-sided $p$ values of less than 0.05 to be statistically significant. Baseline character- istics are reported using descriptive statistics. For the primary outcome, the proportion of patients whose radial-head subluxation was successfully reduced and $95 \%$ confidence intervals (CIs) were determined for each group. The primary analysis was a 95\% CI-based test of noninferiority using a Wilson score CI for the difference between 2 proportions. ${ }^{21}$ To determine if there was a clustering effect, we estimated the intracluster correlation using analysis of variance. ${ }^{22}$ Group-specific design effects were computed, and an adjusted 95\% CI for the difference between 2 proportions was obtained.

For the secondary outcomes time to normal arm usage and length of stay, we used a 2-sided $t$ test to compare means, and we used a Wilcoxon rank sum test as a sensitivity check. As an additional sensitivity check, we also used a weighted $t$ test, in which the weights were the number of cases of radial-head subluxation each day. ${ }^{23}$ For outcomes that were proportions, point estimates and $95 \%$ CIs were determined, and we used Fisher exact or Pearson $\chi^{2}$ tests, as appropriate, for comparison. We had planned to analyze the nurses' success rates by month, but an insufficient number of patients per month precluded meaningful analysis; we instead used 3-month blocks.

\section{Results}

\section{Patient recruitment and baseline characteristics}

During the study period, 416 children had a discharge diagnosis of radial-head subluxation (Figure 1). Of these, 268 met the criteria for the medical directive and were assigned to receive either physician- or nurse-initiated care, depending on the day. During the study period, more than 1 patient was enrolled on 56 days, 1 patient was enrolled on 120 days, and no patients were enrolled on 182 days. There were 2 cases in which a patient randomized in the physicianinitiated care group received care from a nurse, and 21 patients randomized to the nurse-initiated care group had no reduction attempted or no study form was completed, leaving 245 children in the per-protocol analysis. The study groups were similar, although a higher percentage of injuries were related to an arm pull in the nurseinitiated care group (Table 1).

\section{Radial-head subluxation success rate}

In total, $96.7 \%$ of children (117/121) had their injury successfully reduced by a physician on a physician-treatment day (Table 2), and $84.7 \%$ $(105 / 124)$ of children had their injury successfully reduced by a nurse on a nurse-treatment day. The difference in the proportion of children 
who had successful treatment by the appropriate care provider between the 2 groups was $12.0 \%$ (95\% CI $4.8 \%$ to $19.7 \%$ ); noninferiority of nurse-initiated treatment was not shown.

Our primary analysis did not account for the effect of clustering. When clustering was considered, with an estimated intracluster correlation of 0.27 , the adjusted $95 \% \mathrm{CI}$ for the difference in the proportion successful reductions between the nurse-initiated and physician-initiated groups was slightly wider ( $4.3 \%$ to $19.7 \%$ ) than in the primary analysis. There was no improvement over time in the rate of successful radial-head subluxation in the nurse-initiated group.

Although the medical directive states that nurses should attempt a second reduction after an unsuccessful first attempt, 78.9\% (30/38) of children whose first reduction attempt failed received a second attempt by a nurse (Appendix 2, avail- able at www.cmaj.ca/lookup/suppl/doi:10.1503 /cmaj.131101/-/DC1). Among children for whom nurses explicitly followed the medical directive, $90.5 \%(105 / 116)$ of children had a successful reduction. The difference in the proportion of children who had a successful reduction between the physician-initiated and nurse-initiated care groups for those who explicitly followed the directive was $6.2 \%$ (95\% CI $-0.2 \%$ to $13.2 \%)$. Noninferiority was not demonstrated because the upper limit of the confidence interval was outside of the noninferiority margin.

\section{Other clinical outcomes}

Children in the nurse-treatment group had a statistically and clinically significantly shorter overall length of stay in the emergency department compared with children in the physician-treatment group (Table 2). We found no significant differ-

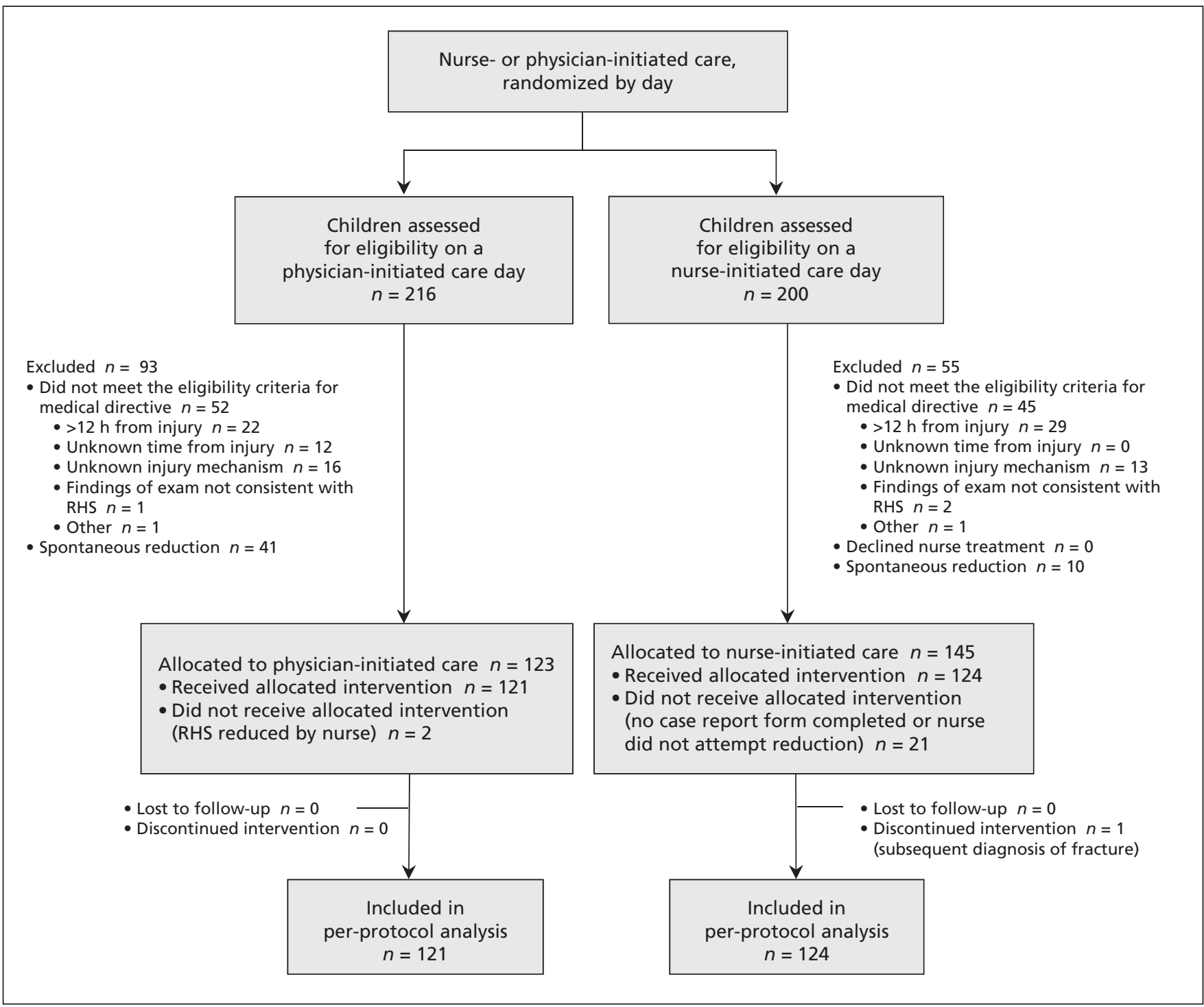

Figure 1: Flow of participants through the study. In this cluster-randomized design, eligible children were assigned to receive nurse- or physician-initiated care based on the day of presentation (cluster). Note: RHS = radial-head subluxation. 
ence between groups in the proportion of children who underwent radiography $(p=0.2)$. One child in the nurse-treatment group who underwent a reduction attempt was subsequently diagnosed with a nondisplaced supracondylar fracture; the child received an above-elbow cast, and there were no complications. Time to normal arm usage was not analyzed because this outcome was only recorded for 70 children.

\section{Technique used for successful reduction}

The reduction technique used was recorded for 91 children who presented on a physician-initiated treatment day (Appendix 2). The successful reduction technique was hyperpronation for $69.2 \%$ of (63/91) of children and supination-flexion for $29.7 \%$ (27/91) children. The success rate of first attempts for physicians was $93.4 \%$ (57/61) for hyperpronation and $83.3 \%$ (25/30) for supination- flexion. The first attempt success rate for nurses (all using the hyperpronation method) was $69.4 \%$ (86/124). Of children who underwent a second attempt by supination-flexion, $63.3 \%$ (19/30) had a successful reduction.

\section{Radial-head subluxation not identified by triage nurses}

During the study period, 13\% (21/145) of children who presented on a nurse-treatment day and for whom radial-head subluxation was diagnosed (and who met the medical directive criteria) had no reduction attempt by a nurse. On review, the triage nurse documented that in 10 cases, the child had a "pulled elbow." The reason a reduction was not attempted is unclear. If we assume conservatively that the triage nurse missed the diagnosis in all 21 cases, $86 \%$ (124 children; $95 \%$ CI $79 \%$ to $90 \%$ ) of radial-head subluxation cases were accu-

Table 1: Characteristics of children included in the analysis

\begin{tabular}{|c|c|c|}
\hline Characteristic & $\begin{array}{l}\text { Nurse-initiated treatment } \\
\qquad n=124\end{array}$ & $\begin{array}{l}\text { Physician-initiated treatment } \\
\qquad n=121\end{array}$ \\
\hline Age, yr, mean \pm SD (range) & $2.4 \pm 1.0(0.4-6.2)$ & $2.2 \pm 1.1(0.3-5.6)$ \\
\hline Female, no. (\%) & $80(64.5)$ & $68(56.2)$ \\
\hline $\begin{array}{l}\text { Time from injury to triage, } \min , * \\
\text { median (IQR) }\end{array}$ & $88(45-153)$ & $103(64-109)$ \\
\hline \multicolumn{3}{|l|}{ Mechanism of injury, no. (\%) } \\
\hline Pull & $118(95.2)$ & $92(76.0)$ \\
\hline Fall & $5(4.0)$ & $19(15.7)$ \\
\hline Other & $1(0.8)$ & $10(8.3)$ \\
\hline
\end{tabular}

Table 2: Clinical outcomes among children assigned to nurse- or physician-initiated radial-head subluxation

\begin{tabular}{|c|c|c|c|}
\hline \multirow[b]{2}{*}{ Outcome } & \multicolumn{2}{|c|}{ No. $(\%)^{*}$ of children } & \multirow[b]{2}{*}{$\begin{array}{l}\text { Difference, } \% \\
(95 \% \mathrm{Cl}) \\
\text { or } p \text { value }\end{array}$} \\
\hline & $\begin{array}{c}\text { Nurse-initiated } \\
\text { treatment } \\
n=124\end{array}$ & $\begin{array}{l}\text { Physician-initiated } \\
\text { treatment } \\
n=121\end{array}$ & \\
\hline $\begin{array}{l}\text { Successful reduction by randomized } \\
\text { care provider }\end{array}$ & $105(84.7)$ & $117(96.7)$ & $\begin{array}{c}12.0 \%(4.8 \% \\
\text { to } 19.7 \%)\end{array}$ \\
\hline Successful reduction by physician & $16(12.9)$ & $117(96.7)$ & \\
\hline $\begin{array}{l}\text { Length of stay in the emergency } \\
\text { department, min, } \dagger \text { median (range) }\end{array}$ & $50(32-82)$ & $105(63-150)$ & $\begin{array}{l}55 \\
<0.001 \ddagger\end{array}$ \\
\hline $\begin{array}{l}\text { Reduction attempted, fracture } \\
\text { subsequently diagnosed }\end{array}$ & $1(0.8)$ & 0 & \\
\hline Underwent radiography & $8 / 124(6.5)$ & $14 / 121(11.6)$ & $0.2 \S$ \\
\hline $\begin{array}{l}\text { *Unless otherwise stated } \\
\text { tLength of stay data was missing for } 2 \text { childre } \\
\text { tWilcoxon Rank Sum test. } \\
\text { §Z test. }\end{array}$ & & & \\
\hline
\end{tabular}


rately identified. If we assume the triage nurse did not miss the diagnosis in the 10 cases for whom "pulled elbow" was recorded, 92\% of radial-head subluxation cases were correctly identified (134 children; $95 \%$ CI $87 \%$ to $96 \%$ ).

\section{Interpretation}

We found an overall success rate for radial-head subluxation of $84.7 \%$ among nurses, which was inferior to the success rate among physicians. In cases where nurses explicitly followed the medical directive, $90.5 \%$ were successfully reduced, although noninferiority was not demonstrated. Nurses accurately identified and reduced radialhead subluxation in most cases. Children in the nurse-treatment group had a shorter length of stay compared with children in the physiciantreatment group, spending an average of $55 \mathrm{~min}$ utes less in the emergency department.

Although nurses reduce radial-head subluxation in some institutions,${ }^{24}$ there have been no studies reporting their success. In our study, no safety concerns were identified. Only 1 child with a fracture was misidentified as having radial-head subluxation; this is lower than the published rate of about $5 \%$ of radial-head subluxation cases being misidentified by physicians. ${ }^{4,6}$ Our length-of-stay data compares favourably to our institution's previous review, which found that patients with radial-head subluxation spend an average of 1.3 hours in the emergency department and that $40 \%$ wait more than 2 hours. $^{8}$ The overall proportion of children who underwent radiography was lower in our study than in other previous studies. ${ }^{8,25}$

It is unclear why the nurses in our study were not as successful as physicians at reducing radialhead subluxation, but it likely relates to experience with the procedure. The medical directive required that nurses first use the hyperpronation technique, and the success of this technique was about $70 \%$, which is less than the reported physician success of $80 \%-95 \% .{ }^{4,67,725}$ In about half of the cases, if the nurse's initial reduction attempt failed, no further attempt was made. When the medical directive was explicitly followed and 2 reductions were attempted if required, the success rate among nurses increased to about $90 \%$.

Although we anticipated that the success rate among nurses would increase over time, we did not observe such an increase during the study period. Our centre has over 60 triage nurses, and given that, on average, less than 1 child presents with radial-head subluxation per day, it is unlikely that any nurse cared for more than a few patients. Over a longer time frame, we would anticipate an increase in nursing success as indi- vidual nurses gain more exposure and comfort with the procedure. Even if the success rates do not increase over time, nurse-initiated treatment does not preclude reduction by a physician or reduce the overall success rate. Given that the overall length of stay in the emergency department was shortened, reduction initiated by triage nurses has the potential to add important value to efficiency in the emergency department.

\section{Limitations}

In our study, a high proportion of children with radial-head subluxation were not eligible for the medical directive because of prolonged time from injury and an unknown mechanism of injury. We did not measure pain associated with the procedure, and this may have been different between the nurse- and physician-treatment groups. We did not randomly allocate individual patients to a treatment group; instead, we used a cluster-randomized strategy, in which care providers were randomized by day, and the allocation was not blinded. As a result, nurses may not have attempted reductions that they perceived to be difficult.

The broader applicability of this protocol may be limited by the high level of nursing experience at our centre and the single centre design. Conversely, the inferiority of nurse-initiated reductions may be because of factors unique to our emergency department. Implementation of the clinical protocol at other sites would determine if the inferiority is consistent. Parents, however, can reduce radial-head subluxation after receiving instruction via telephone ${ }^{26}$ suggesting that experience may not limit applicability.

\section{Conclusion}

The rate of successful radial-head subluxation was lower among nurses than among physicians in our study. However, children who received nurse-initiated care spent less time in the emergency department. Task-shifting in health care involves trade-offs. Our study provides an informed choice between an immediate treatment that works 7 times out of 10 and a delayed treatment that works 19 times out of 20.

\section{References}

1. Schutzman SA, Teach S. Upper extremity impairment in young children. Ann Emerg Med 1995;26:474-9.

2. Salter RB, Zaltz C. Anatomic investigation of the mechanism of injury and pathological anatomy of "pulled elbow" in young children. Clin Orthop Relat Res 1971;77:134-43.

3. Quan L, Marcuse EK. The epidemiology and treatment of RHS Am J Dis Child 1985;139:1194-7.

4. Macias CG, Bothner J, Wiebe R. A comparison of supinationflexion/flexion to hyperhyperpronation in the reduction of RHSs Pediatrics 1998;102:e10.

5. Schunk JE. RHS: epidemiology and treatment of 87 episodes. Ann Emerg Med 1990;19:1019-23.

6. McDonald J, Whitelaw C, Goldsmith LJ. RHS: comparing two methods of reduction. Acad Emerg Med 1999;6:715-8. 
7. Taha AM. The treatment of pulled elbow: a prospective randomized study. Arch Orthop Trauma Surg 2000;120:336-7.

8. Toupin P, Osmond M, Corell R, et al. RHS: how long do children wait in the ED before reduction? CJEM 2007;9:333-7.

9. Magaret ND, Clark TA, Warden CR, et al. Patient satisfaction in the ED - a survey of pediatric patients and their parents. Acad Emerg Med 2002;9:1379-88.

10. Dinh MM, Enright N, Walker A, et al. Determinants of patient satisfaction in an Australian ED fast-track setting. Emerg Med J 2013;30:824-7.

11. Cooper MA, Lindsay GM, Kinn S, et al. Evaluating Emergency Nurse Practitioner services: a randomized controlled trial. $J$ Adv Nurs 2002;40:721-30.

12. Rhee KJ, Dermyer AL. Patient satisfaction with a nurse practitioner in a university emergency service. Ann Emerg Med 1995;26:130-2.

13. Moser MS, Abu-Laban RB, van Beek CA. Attitude of ED patient with minor problems to being treated by a nurse practitioner. CJEM 2004;6:246-52.

14. deForest EK, Thompson GC. Implementation of an advanced nursing directive for suspected appendicitis to empower pediatric emergency nurses. J Emerg Nurs 2010;36:277-81.

15. Zemek R, Plint A, Osmond $\mathrm{MH}$, et al. Triage nurse initiation of corticosteroids in pediatric asthma is associated with improved ED efficiency. Pediatrics 2012;129:671-80.

16. Qazi K, Altamimi SA, Tamim H, et al. Impact of an emergency nurse-initiated asthma management protocol on door-to-firstsalbutamol-nebulization-time in a pediatric ED. J Emerg Nurs 2010;36:428-33.

17. Karpas A, Hennes H, Walsh-Kelly CM. Utilization of the Ottawa ankle rules by nurses in a pediatric ED. Acad Emerg Med 2002; 9:130-3.

18. Krul M, van der Wouden JC, van Suijlekom-Smit LW, et al Manipulative interventions for reducing pulled elbow in young children. Cochrane Database Syst Rev 2012;(1):CD007759.

19. Committee on Proprietary Medical Products. Points to consider on switching between superiority and non-inferiority. London (UK): European Agency for the Evaluation of Medicinal Products;2000. Available: www.ema.europa.eu/docs/en_GB/document library /Scientific guideline/2009/09/WC500003658.pdf (accessed 2013 Nov. 28).

20. Matilde Sanchez M, Chen X. Choosing the analysis population in non-inferiority studies: per protocol or intent-to-treat. Stat Med 2006;25:1169-81.
21. Newcombe RG. Interval estimation for the difference between independent proportions: comparison of eleven methods. Stat Med 1998;17:873-90.

22. Donner A, Eliasziw M, Klar N. A comparison of methods for testing homogeneity of proportions in teratologic studies. Stat Med 1994;13:1253-64.

23. Kerry SM, Bland JM. Analysis of a trial randomised in clusters. BMJ 1998;316:54.

24. Curtis E. Managing "pulled elbow" in the paediatric ED. Emerg Nurse 2012;19:24-7.

25. Rudloe TF, Schutzman S, Lee L, et al. No longer a "nursemaid's" elbow: mechanisms, caregivers, and prevention. Pediatr Emerg Care 2012;28:771-4.

26. Kaplan RE, Lillis KA. Recurrent nursemaid's elbow (annular ligament displacement) treatment via telephone. Pediatrics 2002;110:171-4.

Affiliations: Department of Pediatrics (Dixon), University of Alberta; Stollery Children's Hospital (Dixon), Edmonton, Alta.; Clinical Research Unit (Barrowman), Children's Hospital of Eastern Ontario Research Institute; Children's Hospital of Eastern Ontario Research Institute (Clarkin, Correll, Osmond, Plint), Ottawa, Ont.; Department of Pediatrics and Emergency Medicine (Osmond, Plint), University of Ottawa, Ottawa, Ont.

Contributors: Andrew Dixon developed the hypothesis and the study protocol. Martin Osmond and Amy Plint provided guidance at all stages of the project and provided critical revision of the submitted manuscript. Chantalle Clarkin and Rhonda Correll were responsible for study implementation, data collection and critical revision of submitted manuscript. Nick Barrowman provided consultation on study design, was responsible for all statistical analysis and data interpretation, and provided critical revision of the submitted manuscript. All of the authors approved the final version submitted for publication.

Funding: Funding for this study was provided by the Phase III Academic Health Science Centre/Alternate Funding Plan Innovation Fund. The funding agency had no role in study design, data interpretation, manuscript preparation or publication decisions. 